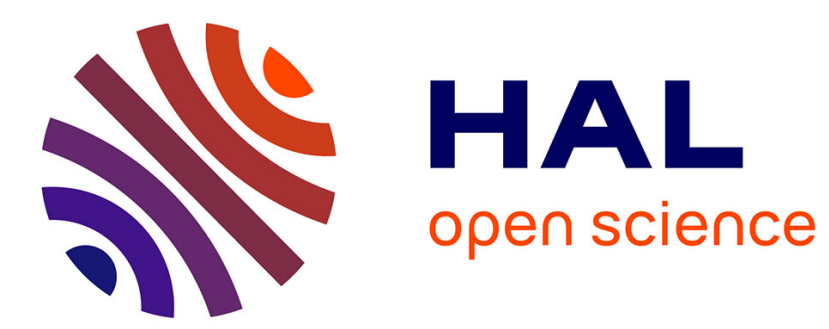

\title{
La construction passive en 'se faire' : une forme concurrente et complémentaire du passif canonique
}

Christel Le Bellec

\section{To cite this version:}

Christel Le Bellec. La construction passive en 'se faire': une forme concurrente et complémentaire du passif canonique. Journal of French Language Studies, 2013, 24 (02), pp.203 - 222. 10.1017/S0959269513000033 . hal-00773574

HAL Id: hal-00773574

https://hal.inria.fr/hal-00773574

Submitted on 7 Mar 2018

HAL is a multi-disciplinary open access archive for the deposit and dissemination of scientific research documents, whether they are published or not. The documents may come from teaching and research institutions in France or abroad, or from public or private research centers.
L'archive ouverte pluridisciplinaire HAL, est destinée au dépôt et à la diffusion de documents scientifiques de niveau recherche, publiés ou non, émanant des établissements d'enseignement et de recherche français ou étrangers, des laboratoires publics ou privés. 


\title{
La construction passive en 'se faire' : une forme concurrente et complémentaire du passif canonique
}

\author{
Christel Le Bellec
}

\section{Résumé}

L'objectif de ce travail est de déterminer la raison d'être de deux formes passives concurrentes en français, à savoir la passive canonique en être suivi du participe passé, comme: Jean a été renvoyé de son travail et la passive en se faire suivi du verbe à l'infinitif, comme : Jean s'est fait renvoyer de son travail. Ainsi, nous examinerons les contraintes aspectuelles qui pèsent sur le passif canonique et qui peuvent obliger le locuteur à utiliser la passive en se faire, ce qui en fait une construction complémentaire. De plus, nous verrons que l'idée traditionnellement répandue dans la littérature selon laquelle la différence entre les deux constructions se situerait au niveau de la responsabilité du référent du sujet, doit être abandonnée au profit d'une différence sémantique reposant sur l'affectation du référent du sujet. 


\section{Introduction ${ }^{1}$}

Dans cet article, nous nous intéresserons aux aspects sémantiques de la construction en se faire d'interprétation passive, comme dans Ils se sont fait cambrioler. Nous tenterons de déterminer la raison de l'existence de deux formes concurrentes du passif en français, à savoir la passive canonique en être suivi du participe passé et la passive en se faire suivi du verbe à l'infinitif. ${ }^{2}$

En premier lieu, nous défendrons l'existence d'une construction en se faire d'interprétation passive à côté d'une construction purement causative (section 1) ; ensuite nous verrons que certaines particularités aspectuelles du passif canonique peuvent contraindre le locuteur à utiliser la passive en se faire au lieu du passif en être (section 2); puis nous examinerons les différences sémantiques entre la passive canonique et la passive en se faire relevant de la responsabilité ou de l'affectation du référent du sujet (section 3) ; et enfin, nous verrons quels sont les types de verbes qui peuvent entrer dans cette construction ainsi que leurs particularités (section 4).

\section{La construction en 'se faire' : passive ou causative ?}

L'équivalence d'interprétation entre les deux constructions suivantes a depuis longtemps été remarquée dans la littérature :

(1) a. Jean s'est fait renvoyer de son travail

b. Jean a été renvoyé de son travail

C'est ce qui a poussé bon nombre d'auteurs à considérer cette construction comme passive (notamment Spang-Hanssen, 1967 et Kupferman, 1995). En effet, elle se construit à partir de verbes transitifs dont le premier argument est le plus souvent un $\mathrm{Agent}^{3}$, qui est optionnellement réalisable en un complément en par, comme dans la construction passive canonique : Jean s'est fait renvoyer/ a été renvoyé de son travail (par son patron); de plus le référent du sujet

\footnotetext{
${ }^{1}$ Nous remercions les relecteurs anonymes pour leurs nombreuses remarques qui ont permis d'améliorer cet article.

2 D'autres constructions jouent un rôle similaire à la passive en se faire, telles que la construction en se voir (voir Bat-Zeev Shyldkrot, 1981 et 1997 ; et François, 2001) et la construction en se laisser (voir Bat-Zeev Shyldkrot, 1999).

${ }^{3}$ Nous verrons plus loin (section 4) que des verbes non agentifs peuvent aussi figurer dans cette construction.
} 
de cette construction est affecté par l'action, dont il est le Patient, à l'instar de la construction passive canonique ${ }^{4}$.

Toutefois, sa forme singulière, composée d'une particule réflexive et du verbe faire, la rend homonyme de la construction causative réfléchie, comme :

(2) Je ne parviens pas à me faire rire

Dans cet exemple, le verbe rire étant un verbe intransitif, la construction résultante ne peut donner lieu à une construction passive, on a donc forcément l'interprétation causative réfléchie ; comme l'indique Novakova (2008: 6), on y trouve un petit nombre de verbes comme: se faire avorter/bronzer/maigrir/suer/vomir, etc.

Ainsi, nous commencerons par examiner les critères qui nous permettent de distinguer entre une construction causative et une passive; puis nous aborderons la grammaticalisation de la construction en se faire et enfin nous étudierons l'ambiguïté de certaines constructions en se faire qui peuvent recevoir l'une ou l'autre interprétation.

\subsection{Deux constructions bien distinctes}

Contrairement à Tasmowski \& van Oevelen (1987), Kupferman (1995) montre que la construction passive en se faire est singulière et qu'elle doit relever d'un traitement distinct de la construction causative réfléchie. C'est également l'idée que nous partageons et que nous tâcherons de défendre dans ce qui suit.

Ainsi, d'après Kupferman (1995), il y a deux constructions en se faire bien distinctes: d'une part la construction causative réfléchie, où le sujet est un 'instigateur' et/ou un Agent et où le verbe faire a un sens causatif, comme dans Elle se fait rire; d'autre part, la construction passive, non causative, où le sujet est un Patient.

Kupferman distingue ces deux constructions notamment par le type de verbes qui peuvent entrer dans celles-ci. Ainsi, on trouve la construction causative réfléchie avec les verbes intransitifs, puisque les verbes intransitifs n'entrent pas dans la construction passive, comme : Elle se fait bronzer, ou bien avec des verbes statifs, comme: Pierre s'est fait connaître du grand public, où dans les deux cas, le sujet de ces constructions est un Agent (ou instigateur) et en même temps un Patient car il y a réflexion du procès sur le sujet, grâce à la présence de la particule réflexive ${ }^{5}$.

Une preuve de l'existence d'une construction passive en se faire en opposition à une construction causative, est que l'on ne peut remplacer le sujet

\footnotetext{
4 Précisons néanmoins que le sujet du passif est un Patient dans la plupart de ses emplois, mais le passif canonique accepte également des verbes non agentifs, comme connaître, voir, etc. dans: Il est connu de tous; Il a été vu par tout le monde.

5 L'interprétation causative réfléchie est aussi possible avec des verbes agentifs, comme nous le verrons dans la sous-section suivante.
} 
par un tiers participant qui serait responsable du procès (tout en conservant le sens de la phrase initiale), comme en (3), ce qui est en revanche possible pour la construction causative, comme en (4), puisqu'elle implique deux rôles sémantiques, l'Agent et le Patient qui peuvent être distribués sur deux arguments référentiellement distincts (exemples de Kupferman, 1995 : 69) :

(3) a. Elle s'est fait agresser par Sam

b. *On l'a fait agresser par $\mathrm{Sam}^{6}$

(4) a. Elle s'est fait rire

b. On l'a fait rire

Néanmoins, la caractérisation que Kupferman propose de cette construction passive occulte toute différence sémantique entre la passive en se faire et la passive canonique et laisse ainsi supposer que les deux constructions sont synonymes. Dans les sections 2 et 3 , nous nous attacherons à l'examen des différences sémantiques et verrons à quels niveaux elles se trouvent, car il serait surprenant que deux formes concurrentes dans la langue soient exactement synonymes. Mais avant cela, nous allons examiner le semi-auxiliaire se faire passif face au semi-auxiliaire (se) faire causatif, au niveau sémanticosyntaxique, afin de mieux comprendre ce qui les distingue.

\subsection{La grammaticalisation du semi-auxiliaire 'se faire'}

D'après Kurylowicz (1965), la grammaticalisation implique le passage d'une catégorie lexicale à une catégorie grammaticale, ou d'une catégorie déjà grammaticale à une unité plus grammaticale. Partant de la construction causative réfléchie, comme: Elle se fait vomir après chaque repas, on passe d'un sens plus ou moins concret (la causation réflexive) à un sens plus abstrait (la passivation), comme dans : Il s'est fait poignarder en plein jour, dont le rapport avec le verbe faire lexical est encore moins sensible, voire inexistant.

Ainsi, le semi-auxiliaire se faire passif serait le résultat d'un processus de grammaticalisation ; plus spécifiquement, il serait le fruit d'une réanalyse, c'està-dire d'une modification de sa structure sous-jacente, consistant en la fusion de deux opérateurs : le verbe faire et la particule réflexive $(($ faire lexical $\rightarrow)$ faire causatif + se réflexif $\rightarrow$ se faire passif). On passe donc d'un élément lexical (au sens de 'fabriquer') à un élément grammatical (à sens causatif), puis de deux éléments grammaticaux combinés entre eux à un nouvel ensemble grammatical dont les deux composants ont subi une désémantisation (perte du sens de causation + perte du sens réflexif). Cette désémantisation des opérateurs

${ }^{6}$ Cette phrase serait acceptable si l'on envisageait le référent de on comme un «mafieux » par exemple, en revanche, elle est inacceptable si on la compare à une phrase passive canonique, comme : Elle a été agressée par Sam, où le référent du sujet ne porte que le rôle de Patient, contrairement à Elle s'est fait rire qui porte les rôles d'Agent et de Patient. 
causatif et réflexif implique une réinterprétation de la construction consistant à attribuer une valeur passive à l'ensemble ainsi constitué.

Néanmoins, la construction en se faire d'interprétation causative n'a pas disparu, elle cohabite dans la langue aux côtés de la construction passive, c'est pourquoi il peut y avoir parfois une certaine ambiguité dans l'interprétation de la forme en se faire; c'est ce que nous allons voir dans la sous-section suivante. Mais avant cela, nous nous intéresserons aux raisons qui font que la construction en se faire aurait dérivé vers une telle fonction. Nous verrons qu'il y a deux principales raisons à cela: la première est d'ordre sémanticosyntaxique et la seconde d'ordre expressif.

En effet, la construction causative, comme : Elle s'est fait ramener par un taxi, outre le fait d'avoir un sens causatif (= elle a fait en sorte qu'un taxi la ramène), présente également un sens passif car la particule réflexive transmet le rôle de Patient au sujet de la construction (= elle a été ramenée par un taxi) en plus de son rôle de Causateur. Par la suite, le sens de la construction aurait dérivé vers des situations sans Causateur, comme Elle s'est fait attaquer par un chien à côté de Elle a été attaquée par un chien, où le sujet n'a plus que le rôle de Patient, entrant donc en concurrence avec le passif canonique.

De plus, l'utilisation de la construction en se faire, pour des emplois passifs, s'explique par le besoin d'expressivité (Meillet, 1912 ; Frei, 1929) ou encore le besoin d'« extravagance » (Haspelmath, 1999), qui consiste pour le locuteur à se faire remarquer, à attirer l'attention de l'interlocuteur sur sa façon de parler, donc à dire les choses de façon plus originale, plus frappante. Ce phénomène est lié, selon Frei, à la loi de l'usure :

«Plus le signe est employé fréquemment, plus les impressions qui se rattachent à sa forme et à sa signification s'émoussent. Du point de vue statique et fonctionnel, cette évolution est contrebalancée par un passage en sens inverse: plus le signe s'use, plus le besoin d'expressivité cherche à le renouveler, sémantiquement et formellement. » (Frei, 1929 : 233)

En effet, la construction passive canonique s'est certainement avérée trop neutre pour exprimer certaines situations à sens passif, ce qui l'a conduite à être substituée par le passif en se faire, qui est plus "expressif » et " extravagant », dans la mesure où il renouvelle l'expression de la passivité, puisqu'en effet, les verbes pris au sens figuré sont plus naturels dans la construction en se faire que dans la passive canonique (voir partie 4.3). L'utilisation de la construction en se faire est alors privilégiée pour dire les choses de façon plus originale et métaphorique, comme dans : Il s'est fait rouler par son patron au lieu de: Il a été roulé par son patron (cette dernière pouvant donner lieu à deux interprétations : l'une au sens propre et l'autre au sens figuré). 


\subsection{De l'ambiguïté de la construction en 'se faire'}

Alors que les verbes intransitifs enchâssés dans la construction en se faire n'ont qu'une interprétation possible: la causative réfléchie, les verbes transitifs peuvent avoir deux lectures : l'une passive, l'autre causative réfléchie.

Avec les verbes de perception et de cognition, comme voir, remarquer, connaître, etc., les deux interprétations sont possibles, selon que le sujet est agentif ou non. En effet, l'adjonction de constituants adverbiaux peut nous induire sur l'une ou l'autre interprétation; ainsi, il y a interprétation passive dans (5a) puisqu'il n'y a aucune intention de se faire remarquer de la part du référent du sujet, alors que dans (9b) il y a interprétation causative, car l'adverbe exprès met en relief l'intentionnalité du référent du sujet :

(5) a. Elle s'est fait remarquer par tout le monde malgré sa grande discrétion

b. Elle s'est fait remarquer exprès pour faire honte à ses amis

De même avec certains verbes agentifs, tels que tuer, conduire, etc. il peut y avoir interprétation causative-réflexive dans la mesure où le référent du sujet est l'instigateur de l'action (6a) ( $=$ il a fait en sorte qu'un taxi le conduise à la gare) ; alors que ce même verbe peut donner lieu à une interprétation passive (6b) (= on l'a conduit de force au commissariat) :

(6) a. Il s'est fait conduire à la gare par un taxi pour être sûr d'arriver à l'heure

b. Il s'est fait conduire de force au commissariat

Ainsi, trois éléments sont déterminants dans l'interprétation causative réfléchie face à l'interprétation passive :

- le sujet est agentif, il est l'instigateur de l'action représentée par le verbe à l'infinitif, il fait en sorte que l'événement ait lieu et à ce titre, il a un double rôle sémantique: instigateur et patient ( $c f$. exemples $5 \mathrm{~b}$ et $6 \mathrm{a}$ ), contrairement au passif où le sujet porte uniquement le rôle de patient $(5 \mathrm{a}$ et $6 b)$;

- la particule réflexive joue pleinement son rôle réflexif : l'action du verbe à l'infinitif se retourne sur le référent du sujet, c'est pourquoi ce dernier acquiert le rôle de patient en plus de celui d'instigateur, alors que dans la passive, la particule réflexive n'a plus aucun rôle sémantique, elle est transparente sémantiquement;

- le verbe faire a un sens causatif, dans la mesure où le référent du sujet fait en sorte que l'événement ait lieu, c'est ce qui lui permet d'acquérir le rôle d'instigateur, mais ce même verbe est transparent sémantiquement dans une interprétation passive. 


\section{L'aspect de la construction passive en 'se faire'}

Il est connu que les deux constructions ne sont pas interchangeables dans tous les contextes ou bien que l'une est plus naturelle que l'autre avec certains verbes. Ceci est le signe d'une différence entre les deux constructions, en effet l'exemple (7a) est bien plus naturel que l'exemple (7b) qui semble plus difficile à accepter dans ce contexte :

(7) a. Lorsqu'il ne va pas à l'école, ou au travail, il se fait enguirlander (...) (Roland Coenen, Eduquer sans punir, 2002)

b. ?Lorsqu'il ne va pas à l'école, ou au travail, il est enguirlandé (...)

Nous allons examiner cette distinction au niveau aspectuel, en commençant par considérer l'aspect de la passive canonique, puis nous examinerons le comportement des deux passives avec les périphrases aspectuelles et avec différents temps verbaux, et enfin, nous verrons en quoi les deux constructions peuvent être complémentaires.

\subsection{L'aspect de la passive canonique}

Le fait que la construction passive en être implique un changement d'aspect par rapport à son homologue actif est un fait largement répandu dans la littérature sur le passif. Cette modification aspectuelle consiste pour le passif à décrire un état résultant du procès alors que son homologue actif décrit le procès en cours de réalisation (Carlier, 2002 ; Helland, 2002 ; Muller, 2000) :

(8) a. Le sommelier sert le vin

b. Le vin est servi

Ce changement aspectuel a lieu exclusivement avec des verbes qui présentent du point de vue de l'aspect lexical ou Aktionsart, un procès télique, puisque l'interprétation d'un état résultant du participe passé n'est disponible que si l'action aboutit à un état achevé stable (Helland, 2002 et Carlier, 2002). Dans (8a), la phrase active évoque un procès en cours, alors que (8b), qui en est l'équivalent au passif, marque l'état résultant de ce procès, l'aspect accompli. Cependant, la présence du complément d'agent dans la phrase passive, peut parfois conserver l'idée d'un procès, d'où l'opposition entre 'passif processif' et 'passif adjectival ou d'état' (Helland, 2002, Muller, 2000) :

(9) a. La maison est construite par une firme suédoise (= passif processif) (Muller, 2000)

b. La maison est construite en briques (= passif adjectival/état résultant) (ibid.) 
En revanche, les verbes atéliques, qui marquent donc un état ou une activité, ne diffèrent pas, sur le plan aspectuel, de leur contrepartie active, comme le montrent les exemples suivants (Carlier, 2002: 42):

(10) a. Tout le monde apprécie Pierre

b. Pierre est apprécié de tout le monde

(11) a. Marie aide Pierre

b. Pierre est aidé par Marie

À l'instar de Carlier (2002 : 43), l'insertion de la locution être en train de peut être utilisée comme test afin de révéler le caractère processif de la situation évoquée. En effet, ce test fait apparaître que le passif canonique est moins naturel qu'avec le passif en se faire, où la locution s'insère tout naturellement, car elle maintient le caractère dynamique du verbe processif :

(12) a. Regardez! Il est en train de se faire frapper par sa femme!

b. ?Regardez! Il est en train d'être frappé par sa femme!

La différence aspectuelle que l'on observe entre les deux constructions passives s'explique notamment par le fait que l'une a recours au participe passé pour se former, tandis que l'autre a recours à l'infinitif; le participe passé étant un marqueur de l'accompli (Carlier, 2002: 45) et l'infinitif un marqueur de l'inaccompli.

\subsection{Les périphrases aspectuelles}

Selon Spang-Hanssen (1967: 141), la construction en se faire remplace la passive canonique «quand cette dernière ne peut être employée pour marquer une action en cours » et «avec la construction en se faire, on exprime plus nettement l'aspect inchoatif ou l'idée d'un processus ». En effet, lorsque le verbe aller est employé dans une périphrase verbale temporelle, le passif canonique est plus difficile (13b) et (14b), puisque la tournure requiert normalement de voir l'entrée dans le procès, ce que ne peut exprimer la passive canonique, qui présente l'état résultant du procès; c'est donc la passive en se faire qui est utilisée dans de tels contextes, afin de suppléer à cette restriction aspectuelle :

(13) a. Tu vas te faire gronder si tu continues

b. ?Tu vas être grondé si tu continues

(14) a. Si tu n'es pas prudent tu vas te faire renverser par une voiture

b. ?Si tu n'es pas prudent tu vas être renversé par une voiture

Novakova (2008: 7) remarque une fréquence élevée de la construction en se faire après les périphrases aspectuelles exprimant les phases du procès, comme : commencer à, être en train de, finir de, etc. En effet, étant donné que le passif canonique renvoie à des procès accomplis, il est difficilement compatible 
avec les différentes phases du procès. En revanche, la construction en se faire est compatible avec toutes les phases puisqu'elle renvoie à des procès inaccomplis ou en cours de déroulement. De même après les verbes de perception (voir, entendre, regarder), la présence de se faire est préférable au passif canonique, en raison des propriétés aspectuelles de la construction qui renvoient surtout à des procès en cours de déroulement (Novakova, ibid. : 9) :

(15) a. Nous regardions sur CNN des villes se faire bombarder à la télévision (ibid.)

b. ?Nous regardions sur CNN des villes être bombardées à la télévision (ibid.)

\subsection{Le temps de la construction}

Lorsque Gaatone (1983: 165) présente le contraste entre les deux exemples suivants, comme étant dû au fait que la passive en se faire présente le référent du sujet comme responsable du procès, contrairement au passif canonique, il s'agit en réalité d'une question de compatibilité du temps avec l'aspect de la construction :

(16) a. Elle est folle. Elle se fera écraser par le tramway. (Danell, 1979 : 62)

b. \#Elle est folle. Elle sera écrasée par le tramway.

En effet, dans (16a), la phrase implique de voir l'entrée dans le procès, alors que dans (16b) on ne voit que l'état résultant de l'action. L'inacceptabilité du second exemple serait donc due à l'aspect inadéquat de cette construction, car cet énoncé implique que l'on prenne en compte l'étape d'entrée dans le procès, ainsi que son déroulement; or le passif canonique n'en présente que le résultat.

L'exemple au passif canonique est toutefois plus acceptable au passé composé (17a), mais reste plus difficile à l'imparfait et au présent (17b) et (17c), car ces deux temps privilégient l'état résultant :

(17) a. Elle a été écrasée par le tramway

b. Elle était écrasée par le tramway

c. Elle est écrasée par le tramway

Néanmoins, avec la construction en se faire, il n'y a pas de tels contrastes d'acceptabilité selon le changement de temps, car elle est apte à présenter les différentes étapes du procès :

(18) a. Elle s'est fait écraser par le tramway

b. Elle se faisait écraser par le tramway

c. Elle se fait écraser par le tramway 
On voit à travers ces exemples que le temps de la construction peut également contribuer à faire prendre au passif une valeur d'état résultant ou bien au contraire privilégier la valeur processive de l'actif correspondant. Ainsi, le passé simple et le passé composé maintiennent cette valeur processive, alors qu'à l'imparfait, au présent et au futur, le passif tend à faire prendre une valeur d'état résultant à la construction. Comme l'indique Carlier (2002 : 58), le passé simple, qui offre une vision globale de la situation et l'appréhende comme pourvue d'une borne initiale et d'une borne finale, présente une prédilection pour les verbes processifs téliques, tandis que l'imparfait, qui présente une vision sécante de la situation et fait donc abstraction de ces bornes, n'est pas prédisposé à entrer en combinaison avec un verbe processif télique.

\subsection{Une complémentarité aspectuelle}

Lamiroy (1993) et Lagae (2002) soutiennent qu'il y a complémentarité aspectuelle entre le passif canonique et le passif pronominal, qui fait que ce dernier s'associe mieux avec l'aspect inaccompli et corollairement le passif canonique avec l'aspect accompli ${ }^{7}$ :

(19) a. ?Cela est raconté partout (Lamiroy, 1993 : 66)

b. Cela se raconte partout

(20) a. La voiture est garée devant la porte (Lagae, 2002 : 142)

b. La voiture se gare devant la porte

Nous ajouterons qu'il y a complémentarité aspectuelle entre le passif en se faire et le passif en être, comme nous l'avons vu dans cette section, venant s'ajouter en triangle à l'hypothèse d'une complémentarité entre passif canonique et passif pronominal (voir figure 1 ci-dessous).

En effet, la construction passive en se faire d'aspect inaccompli permet, sous certaines conditions, de suppléer aux restrictions du passif canonique lorsque celui-ci ne peut évoquer l'idée d'un processus, notamment lorsqu'il marque l'état résultant, comme nous l'avons vu précédemment. Passif en se faire et passif pronominal sont tous deux propices à l'expression de l'aspect inaccompli en raison de leur formation morphologique : le premier est formé du verbe à l'infinitif, étant l'expression de l'aspect inaccompli, le second est constitué d'un verbe conjugué, le plus souvent au présent, temps de l'inaccompli par excellence également. Le passif canonique, en revanche,

\footnotetext{
${ }^{7}$ Lagae (2002) montre qu'en réalité il n'y a que très peu d'occurrences au passif pronominal qui évoquent l'idée d'un procès en cours, car généralement ces constructions, comme La voiture se gare devant la porte ou Les légumes se pèsent véhiculent des valeurs habituelles, normatives ou génériques, plutôt que d'évoquer un procès en cours, comme le ferait leur homologue actif. Ainsi, d'après cet auteur, les propriétés spécifiques du passif pronominal vont plutôt dans le sens d'une spécialisation que d'une complémentarité (Lagae, 2002 : 135).
} 
s'oriente vers l'aspect accompli en raison notamment de la présence du participe passé dans sa formation, indicateur de l'aspect accompli.

De plus, la passive en se faire s'est spécialisée dans la topicalisation d'arguments animés ${ }^{8}$, comme nous le verrons dans la sous-section 3.3 (par exemple : Le facteur s'est fait mordre par un chien par opposition à * Le colis s'est fait livrer par le transporteur au lieu de Le colis a été livré par le transporteur), tandis que la construction moyenne consiste le plus souvent à topicaliser des arguments inanimés (par exemple : Les vêtements en laine se lavent à froid par opposition à ${ }^{*}$ Les ouvriers se payent à l'heure au lieu de Les ouvriers sont payés à l'heure). Le passif canonique est, quant à lui, indifférent au niveau du caractère $+/$ - animé du référent du sujet.

Voici le schéma récapitulant cette triple complémentarité :

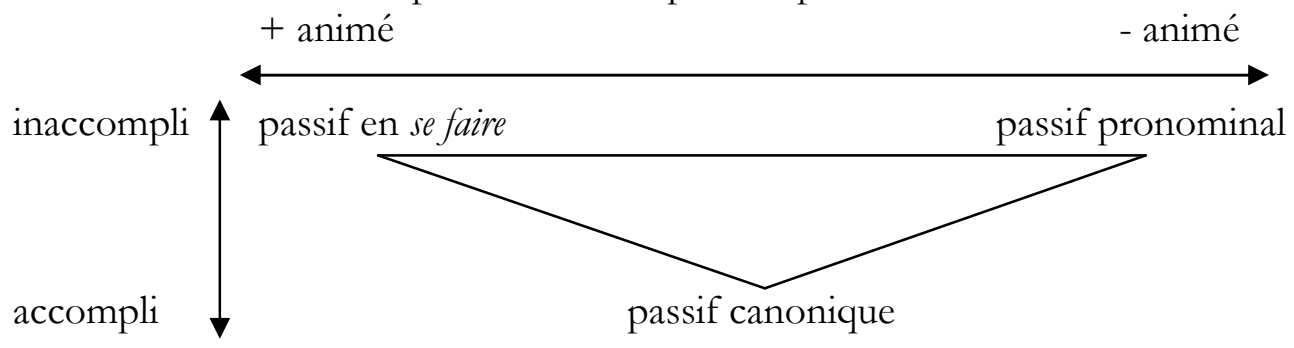

Figure 1 : complémentarité aspectuelle entre trois passifs

\section{Le référent du sujet : responsable ou affecté ?}

\subsection{La traditionnelle " responsabilité " du référent du sujet}

Selon Tasmowski \& van Oevelen (1987) et Donaldson (1973), le référent du sujet de la construction en se faire participe à l'action d'une certaine façon, ce qui justifierait la présence de faire (comme verbe causatif). Tasmowski \& van Oevelen ajoutent que si le sujet de faire et l'objet du verbe enchâssé sont coréférentiels, alors il peut y avoir interprétation passive, ce qui fait de l'interprétation passive un sous-type de la construction 'causative réfléchie'; c'est pourquoi, d'après ces auteurs, le référent du sujet de cette construction a toujours une certaine responsabilité dans l'action. On peut illustrer leur point de vue grâce à l'exemple suivant :

(21) L'accusé (Cause+Patient) $)_{\mathrm{i}}$ s' (Pat) $)_{\mathrm{i}}$ est fait condamner (= l'accusé est responsable de sa propre condamnation)

Nous arguerons, au contraire, que le référent du sujet de cette construction n'est pas causatif, il n'est pas plus responsable dans la passive en se faire que

8 À l'exception des exemples du type : Un bruit sourd se fit entendre, que nous aborderons dans la section 3 . 
dans la passive canonique : L'accusé a été condamné, car il ne reçoit pas le rôle de Cause (ou Causateur), normalement transmis par le verbe faire, étant donné que l'ensemble se faire est interprété comme un semi-auxiliaire de passivation (voir section 1): le réflexif se n'a plus d'interprétation réfléchie et le verbe faire est transparent sémantiquement; seul le rôle de Patient est attribué au sujet de la construction :

\section{(22) L'accusé (Patient) $)_{i} s_{i}^{\prime}$ est fait condamner}

Comme l'affirment certains auteurs (Kokutani, 2005 : 214, à la suite de Le Goffic, 1993, de même que Veecock, 2008: 2210), cette notion de «responsabilité » imputée au sujet de la construction n'est pas une responsabilité ordinaire, mais est à entendre comme une sorte de «responsabilité ontologique», c'est-à-dire que le référent du sujet est responsable parce qu'il est là, par sa simple présence.

Or, il est assez difficile de concevoir une responsabilité de par la simple présence du référent du sujet, même pour une phrase comme (23a), et quand bien même cette arrestation serait la conséquence des actes du référent du sujet, le voleur n'a pas plus de responsabilité dans (23a) que dans (23b) :

(23) a. Le voleur s'est fait arrêter par la police

b. Le voleur a été arrêté par la police

En effet, si l'on introduit des indices de responsabilité, on voit que rien ne distingue (24a) de (24b), où dans les deux cas le voleur a, de toute évidence, une responsabilité dans son arrestation, ni (25a) de (25b), où Marc ne peut être considéré comme responsable du cambriolage dans la mesure où il a pris certaines précautions pour s'en prémunir :

(24) a. Le voleur s'est fait arrêter par la police à cause de ses nombreuses récidives

b. Le voleur a été arrêté par la police à cause de ses nombreuses récidives

(25) a. Marc a installé une alarme dans sa maison, mais il a quand même été cambriolé

b. Marc a installé une alarme dans sa maison, mais il s'est quand même fait cambrioler

Nous verrons ci-après que ce qui est en jeu ici, ce n'est pas la responsabilité tant soutenue dans la littérature, mais plutôt le degré d'affectation plus ou moins grand du référent du sujet.

\subsection{L'affectation du référent du sujet}

La valeur aspectuelle que prend la passive en se faire, a pour conséquence une interprétation particulière du référent du sujet, qui tend à prendre en plus de son rôle de Patient une valeur d'entité particulièrement affectée par le procès, 
ce qui va le distinguer encore une fois, mais de façon plus radicale, de la passive canonique.

Ainsi, dans la construction en se faire, le référent du sujet est présenté comme grandement affecté du fait que l'on expose l'ensemble du procès en cours de déroulement, tandis que dans la passive canonique, le référent du sujet est normalement affecté par un procès dont on ne voit que le résultat, comme si la présentation du résultat du procès en diminuait l'impact sur le patient et qu'au contraire la visualisation d'un procès en train de s'écouler en accentuait l'affectation.

De plus, le fait que cette construction soit plus fréquemment utilisée pour des procès "désagréables »" (cf. Gaatone, 1983) et rarement utilisée pour des sujets inanimés corrobore sa propension à y présenter un patient grandement affecté, là où le passif canonique est indifférent (puisqu'utilisé tant pour des sujets animés qu'inanimés et avec tout type de procès).

Cet effet de sens nous permet ainsi d'expliquer pourquoi la phrase (26b) ci-dessous au passif canonique est déviante. En effet, elle n'implique pas forcément que le référent du sujet soit entièrement brûlé au point d'en mourir, alors que la passive en se faire en (26a) évoque l'idée d'une mort consécutive à la destruction par le feu :

(26) a. Le démon me souffle que les fidèles qui se font brûler ne ressusciteront pas (Biancotti, H., Sans la miséricorde du Christ, 1985, p. 345) b. ?Le démon me souffle que les fidèles qui sont brûlés ne ressusciteront pas.

Il semble donc que la passive en se faire implique une plus grande implication du référent du sujet dans le procès, une réelle affectation qui n'est pas aussi forte avec la passive canonique, qui est ressentie comme plus neutre quant à l'affectation. Cette construction donne également plus d'expressivité à la phrase et marque une certaine empathie du locuteur envers le référent du sujet, comme nous le verrons dans la sous-section suivante.

Cette implication du référent du sujet dans le procès peut être en bien ou en mal, c'est-à-dire que si le verbe dénote une action désagréable, il en sera encore plus désagréable pour l'entité affectée (ex. 27a et 28a), et si le procès est agréable, il en sera également plus agréable (ex. 29a et 30a) :

(27) a. Il se faisait piétiner sans cesse

b. Il était piétiné sans cesse

(28) a. Elle s'est fait gifler par son professeur

b. Elle a été giflée par son professeur

${ }^{9}$ Sur un corpus littéraire de 80 occurrences du passif en se faire, nous ne recensons que trois occurrences de situations pouvant être considérées comme «agréables » pour le référent du sujet. Le reste correspondant à des situations considérées comme « désagréables ». 
(29) a. On n'a jamais entendu dire qu'un type puisse se faire toucher par la Grâce sur le parking d'un supermarché (Djian, P. 37² le matin, 1985, p. 236)

b. On n'a jamais entendu dire qu'un type puisse être touché par la Grâce sur le parking d'un supermarché

(30) a. Nous nous sommes fait accueillir comme des rois

b. Nous avons été accueillis comme des rois

Cette plus forte implication du référent du sujet dans le procès fait que cette construction est souvent employée pour dénoter des procès où est en jeu la vie du référent du sujet, sa santé, une partie de son corps, ses biens, son intégrité, sa dignité, etc., tout ce qui peut impliquer un changement important, changement qui le touche profondément ${ }^{10}$ (ce qui a conduit Gaatone : 1983 à parler de verbes dénotant des actions désagréables), alors que la passive canonique est neutre dans ce domaine-là.

On trouve ainsi de nombreux exemples où l'intégrité (physique ou psychique) du référent du sujet est grandement en péril :

(31) Il s'est fait tabasser

(32) Il se fit virer par le portier à grands coups de pieds au cul (Benoziglio, J.L., Cabinet portrait, 1980, p.16)

(33) Ils se faisaient chasser à coups de pierre par les bergers (Weyergans, F., Macaire le Copte, 1981, p.71)

(34) Me faire traiter de phallocrate! (Boudard, A., Les Enfants de chcour, 1982, p.80)

Les particularités syntaxiques de la construction en se faire permettent également de présenter une partie du corps comme entièrement affectée par le procès, en fonction d'objet direct ( $c$. exemple 35a), alors que dans la passive canonique (35b), la partie du corps n'est que partiellement affectée, car le complément prépositionnel à la main est un complément de lieu. Il indique donc que la brûlure a eu lieu en un endroit de la main mais non sur l'ensemble de la main comme dans la passive en se faire où ce SN est en position d'objet direct :

(35) a. Il s'est fait brûler la main

b. Il a été brûlé à la main

\subsection{Le caractère +/- animé du sujet}

D'après Tasmowski \& van Oevelen (1987) et Gaatone (1983), l'idée d'une responsabilité du référent du sujet dans le procès donnerait l'explication du fait

\footnotetext{
10 Voir note précédente.
} 
que le causatif pronominal parait exiger un sujet animé, d'où l'agrammaticalité ou l'inacceptabilité des exemples suivants :

(36) *Son piano s'est fait abîmer par les déménageurs (TO, 1987 : 48)

(37) *La proposition s'est fait rejeter par tous les députés (Gaatone, 1983 : 166)

(38) Le pauvre garçon s'est fait rayer de nos listes. \#Il est mort. (TO, 1987 : 47)

(39) a. La victime n'a pas encore pu se faire interroger. \#Elle est toujours dans le coma. (TO, $1987: 47$ )

b. La victime n'a pas encore pu être interrogée. Elle est toujours dans le coma. (ibid.)

Concernant la paire d'exemples (39), la construction en se faire n'est pas naturelle car il n'y a pas d'intérêt à voir le déroulement du procès, 'la victime' étant comateuse, comme la construction en se faire l'indiquerait, seul le résultat est pertinent comme le révèle la phrase passive canonique en (39b).

À la différence de Tasmowski \& van Oevelen et Gaatone, nous arguerons que le fait que les sujets inanimés soient difficilement acceptables ou ont du mal à figurer dans la construction passive en se faire est probablement dû au fait qu'un inanimé peut difficilement être affecté et que par conséquent l'on peut difficilement ressentir de l'empathie pour un inanimé. En effet, on peut éprouver de l'empathie pour les êtres humains, voire pour les animaux, mais difficilement pour les objets. C'est pourquoi on peut dire aisément (40a), mais (40b) est plus distant, plus austère; à propos d'un animal, les deux constructions sont possibles : (41a) fait preuve d'attendrissement et (41b) est plus neutre, sans pitié aucune; et à propos d'un inanimé (42a) est étrange et connote une certaine affection envers la maison, comme si on personnifiait la maison, alors que (42b) est tout à fait ordinaire :

(40) a. Quelle horreur! Elle s'est fait manger par un tigre!

b. Quelle horreur! Elle a été mangée par un tigre!

(41) a. La pauvre gazelle! Elle s'est fait manger par le lion!

b. La gazelle a été mangée par le lion; c'est la loi de la nature

(42) a. ?Ma maison s'est fait repeindre

b. Ma maison a été repeinte

L'exemple (43a) de Labelle est acceptable malgré la présence d'un SN inanimé en position de sujet. L'acceptabilité est ici due à la personnification du référent du sujet grâce à l'adjonction de l'adjectif gentil qui lui attribue des traits humains et qui permet ainsi de présenter une entité plus fortement impliquée dans le procès qu'avec le passif en être (43b) :

(43) a. Notre gentil projet de loi s'est fait adopter dans sa première mouture (Labelle, $2002: 16$ )

b. Notre gentil projet de loi a été adopté dans sa première mouture. 
D’après Novakova (2008: 10), le passif canonique est assez fréquent dans les textes scientifiques, mais le passif en se faire est quasiment absent, en raison du désir d'objectivité requis par ce type de textes. En effet, cela va dans le sens de notre postulat, à savoir que cet évitement est dû au fait que le locuteur ne peut faire mention de la grande affectation d'un actant ni montrer trop d'empathie pour rester le plus objectif possible.

De plus, la construction en se faire se trouve fréquemment dans un registre populaire ou familier ${ }^{11}$, contrairement à la construction en se voir, que l'on trouve plus aisément avec des verbes désignant des actes officiels, comme : Il s'est vu remettre la Légion d'Honneur (François, 2001). En effet, nombreux sont les exemples où la construction en se faire est associée à des verbes familiers, voire grossiers, dans un langage relâché. En voici quelques exemples :

(44) Je suis en train de me faire rouler

(45) Tu vas te faire massacrer!

(46) J'ai réussi à me faire refouler par un homme de Néanderthal et son copain Cromagnon (Manœuvre, P., L'enfant du rock, 1985, p.123)

(47) Ils ont dû se faire engueuler

(48) Bref. Mon pote s'est fait larguer (www.canalplus.fr, épisode du 10/10)

Pour terminer la discussion sur la responsabilité et l'affectation du référent du sujet, nous voulons signaler certains exemples qui ne cadrent avec aucune des deux interprétations, étant donné que le référent du sujet est inanimé. Voici quelques exemples :

(49) Son souffle [d'un sanglier] se faisait entendre de loin comme le bruit sourd des vents séditieux (Fénelon, Les aventures de Télémaque, 1699)

(50) Lorsque la nouvelle se faisait savoir pour la première fois (...).

Ce genre de construction se trouve essentiellement à l'écrit et dans des contextes indiquant le surgissement d'un bruit, d'un son, d'une parole, etc. avec un nombre très limité de verbes, comme entendre et savoir.

Cet emploi est en contradiction totale avec, d'une part, le fait que le référent du sujet soit un animé et, d'autre part, avec le fait de relever d'un registre oral (vs. écrit pour ces exemples). On a bien affaire à une construction passive dans les deux cas (puisqu'elles trouvent leur équivalent au passif canonique: Son souffle était entendu de loin; Lorsque la nouvelle était sue pour la première fois) mais à la différence du passif en se faire que nous avons étudié dans cet article, celui-ci semble figé, car seuls quelques verbes peuvent y figurer.

Son évolution et sa spécialisation aspectuelle sont les mêmes que pour la construction passive en se faire mais on a l'impression que son développement

11 Sur un corpus littéraire de 80 occurrences de passif en se faire, nous recensons une trentaine d'occurrences où se faire est associé à un verbe relevant d'un registre populaire ou familier. 
s'est arrêté à ces quelques verbes pour les entités inanimées, tandis qu'elle a remporté un franc succès pour les sujets animés, dans des contextes oraux.

\section{Les verbes qui entrent dans la construction passive en 'se faire'}

Tous les verbes ne peuvent entrer dans la construction passive en se faire. En effet, nous avons vu que cette construction s'attache à des prédicats verbaux dont le premier argument porte, dans la plupart des cas, le rôle sémantique d'Agent (Kupferman, 1995) qui sera réalisé optionnellement par un complément en par, c'est pourquoi les phrases suivantes présentant non pas un Agent mais une Cause, sont agrammaticales :

(51) *Il s'est fait tuer par son orgueil (Kupferman, 1995 : 67)

(52) Léa s'est fait décoiffer par Max/*par le voyage (ibid.)

D'après Kupferman, cette propriété exclut les verbes statifs de la construction passive en se faire et oriente la lecture de celle-ci vers une construction causative, comme en (53a et b) ; toutefois, la lecture passive reste possible, comme on l'a vu, lorsque le contexte l'impose, comme en (53c):

(53) a. Pierre sait se faire aimer de ses étudiants

b. Pierre s'est fait connaître du grand public en écrivant

c. Pierre se fait remarquer à cause de son nez aquilin

Nous allons voir que cette construction privilégie certains verbes; nous examinerons ainsi le cas des verbes dénotant des actions désagréables (suggéré par l'article de Gaatone, 1983 "Le désagréable en syntaxe »), le cas des verbes ditransitifs et enfin les valeurs au sens propre et figuré de certains verbes.

\subsection{Verbes dénotant des actions désagréables ?}

Il a souvent été noté que la construction en se faire réfère à des actes violents et désagréables (notamment Spang-Hanssen, 1967 et Gaatone, 1983). Tasmowski \& van Oevelen (1987) et Gaatone (1983) estiment que c'est un effet pragmatique, vu que la lecture de la construction causative serait écartée parce qu'il se conçoit mal que le référent du sujet rechercherait volontairement son propre malheur. Or, cette construction ne s'attache pas uniquement à des prédicats dénotant des actes désagréables mais aussi à des prédicats dénotant des actes agréables, comme on l'a vu précédemment et comme le montrent les exemples suivants :

(54) Le quatuor anglais s'est fait acclamer par la salle pleine d'admirateurs (http://info-culture.biz/2012/10/12/)

(55) Il s'est fait séduire par un plat typiquement coréen 
Selon Kupferman, il suffit que le sujet de la construction soit un Patient, qu'il soit affecté par le procès et qu'il subisse un changement d'état, mais que celui-ci soit agréable ou désagréable n'importe pas. Il est vrai que la plupart de ces constructions dénotent des actions désagréables, mais cette prédilection pour ce type d'actions provient du fait que le rôle de Patient grandement affecté exprime plus souvent une affectation en mal qu'en bien.

Spang-Hanssen (1967 : 142) remarque que l'emploi de se faire est la règle lorsqu'on prédit des ennuis à quelqu'un: Tu vas te faire gronder; tu vas te faire prendre, etc. de même lorsque l'on profère des malédictions à la forme impérative du type : va te faire...

Ceci est la conséquence du sens que se faire fait prendre à la construction ; en effet, puisque celle-ci dénote une grande affectation du référent du sujet, il est normal que l'on y retrouve régulièrement des prédicats dénotant des actions désagréables, surtout dans les formulations insultantes, comme aller se faire..., car l'on souhaite que le référent à qui est adressée cette formule soit fortement affecté par la malédiction proférée contre lui. Ceci est également corroboré par l'aspect de cette construction car des tournures au passif canonique, du type : *Va être vu ou *Allez être foutus sont impossibles au regard des formules insultantes : Va te faire voir et Allez vous faire foutre.

\subsection{Les verbes ditransitifs}

Comme l'a notamment souligné Spang-Hanssen, la construction en se faire permet de transformer l'objet indirect de la phrase active en sujet de la phrase passive, ce qui est impossible pour la passive canonique, comme dans :

(56) a. Les riches dames mûres se faisaient voler leurs bijoux par des gigolos argentins (Spang-Hanssen, $1967: 144$ )

b. *Les riches dames mûres ont été volées leurs bijoux

(57) a. Il se fait écraser le pied par un bus

b. *Il est écrasé le pied par un bus

La construction en se faire viendrait donc pallier l'impossibilité qu'a la passive canonique de topicaliser un argument qui reçoit normalement la fonction d'objet indirect ${ }^{12}$. À la différence du passif en se faire que l'on a étudié jusque-là et qui entre en concurrence avec le passif canonique, cette construction est donc supplétive par rapport au passif en être.

On trouve aussi fréquemment la construction en se faire avec des verbes ditransitifs qui appartiennent essentiellement aux verbes de don (ex: se faire offrir/servir), de transfert (ex : se faire livrer/prêter/rembourser) et de dire (ex : se faire conseiller/dédicacer/dicter/notifier) ou encore qui expriment des actes liés au corps

${ }^{12}$ D’après François (2001 : 173), étant donné que le français ne dispose pas de passif formé sur l'objet indirect (comme pour l'anglais), la passive en se faire, ainsi que la passive en se voir permettraient d'y remédier. 
humain (ex : se faire couper les cheveux/épiler les sourcils, etc.) (Novakova, $2008: 5$ ); mais ces constructions sont causatives car le référent du sujet est interprété comme l'instigateur de l'action :

(58) a. Je me suis fait servir une assiette de foie gras

b. Je me suis fait rembourser les frais de scolarité

c. Elle s'est fait arracher une dent par son dentiste

d. Elle s'est fait refaire le nez par le meilleur chirurgien de la ville

À la différence de la construction passive en se faire non ditransitive, celle-ci attribue le rôle sémantique de Destinataire au sujet de la construction (qu'elle soit passive ou causative) car il correspond à un objet indirect dans la construction active (par ex: Des gigolos argentins ont volé les bijoux aux riches dames mûres ou Son dentiste lui a arraché une dent). Quant à l'objet direct, il conserve son rôle de Patient, non plus du seul verbe à l'infinitif comme dans la construction active, mais de la construction entière (puisqu'on a affaire à un complexe verbal : Les dames se les faisaient voler).

\subsection{Sens propre/sens figuré}

L'opposition aspectuelle entre la passive en se faire et la passive canonique permet également d'expliquer le fait que certaines constructions au sens figuré se sont figées, comme : se faire pincer, se faire avoir, se faire rouler, se faire prendre, se faire plumer, etc. au lieu de être pincé, *être eu, être roulé, être pris, être plumé, etc. qui sont moins naturelles, voire agrammaticales, dans le sens figuré qu'elles prennent avec se faire.

Pour ce type de verbes, le passif en être privilégie une lecture au sens propre, même si le sens figuré est également possible, alors qu'avec se faire on a exclusivement un sens figuré, qui provient à la fois de l'effet de sens qu'offre cette construction, à savoir une grande affectation de l'entité à fonction sujet, et du fait qu'elle a pour vocation à exprimer un procès en cours, alors que le passif canonique en exprime l'état résultant.

La passive en se faire permet donc d'évacuer une certaine ambigüité entre deux acceptions d'un même verbe, pris aux sens propre et figuré. Par exemple avec le verbe pincer on peut avoir le sens propre dans la passive canonique ou plus rarement le sens figuré de «arrêter» (59b) et (60b), alors qu'on a exclusivement le sens d'« arrêter» avec la construction en se faire (59a) et l'idée de «saisir en serrant entre ses doigts» est complètement écartée de cette dernière (60a) :

(59) a. Il s'est fait pincer par la police avec de l'ecstasy dans le coffre de sa voiture

b. Il a été pincé par la police avec de l'ecstasy dans le coffre de sa voiture

(60) a. *Il s'est fait pincer au bras par la police pour vérifier son état de conscience

b. Il a été pincé au bras par la police pour vérifier son état de conscience 
De même, la phrase en (61a) est plus naturelle pour signifier qu'il s'est fait tuer, dans la mesure où le verbe descendre s'interprète au sens figuré, ce qui le rend synonyme du verbe tuer et où le référent du sujet est donc grandement affecté, tandis que dans (61b), deux lectures sont possibles : celle où descendre est synonyme de tuer et celle où le tueur à gages l'a descendu à la force des bras (à la cave, par exemple), qui est la lecture privilégiée, une lecture donc au sens propre :

(61) a. Il s'est fait descendre par un tueur à gages

b. Il a été descendu par un tueur à gages

\section{Conclusion}

Nous avons tenté de déterminer la raison d'être de la construction passive en se faire en français, de montrer pourquoi cette forme est en concurrence avec le passif canonique. Nous avons donc soulevé deux raisons essentielles, l'une aspectuelle, l'autre sémantique, qui déterminent les emplois de se faire; le tout étant corroboré par des arguments concernant le type de verbe pouvant entrer dans cette construction.

La première raison qui contraint le locuteur à employer se faire plutôt que la passive canonique (les deux sont en distribution complémentaire, c'est-à-dire que la passive en se faire s'impose là où la passive canonique est impossible ou peu naturelle), concerne les cas où la phrase requiert de voir le procès en cours, alors que la passive canonique exprime l'état résultant du procès. En ce sens, la passive en se faire vient suppléer aux restrictions aspectuelles du passif canonique. De même, pour topicaliser l'argument objet indirect de la structure active, la construction en se faire est supplétive par rapport au passif en être qui lui est impossible.

La seconde raison qui cette fois-ci pousse le locuteur à choisir se faire plutôt que la passive canonique (là où les deux sont généralement possibles, elles sont alors toutes deux concurrentes), concerne les cas où l'être décrit est fortement affecté par l'action et considéré avec empathie par le locuteur. Nous avons vu que c'est pour cette raison que cette construction s'applique essentiellement à décrire des sujets humains et que la traditionnelle « responsabilité » du référent du sujet ne permet pas de justifier l'existence de cette construction.

\section{Références}

Bat-Zeev Schyldkrot, H. (1981). A propos de la forme passive 'se voir + Vinf. Folia Linguistica, 15 : 3-4 : 387-407.

Bat-Zeev Schyldkrot, H. (1997). La grammaticalisation des auxiliaires : le cas de voir. SCOLLA 10 : 205-224.

Bat-Zeev Schyldkrot, H. (1999). Analyse sémantique d'une forme passive complémentaire : se laisser. Langages, 135 : 63-74. 
Carlier, A. (2002). Les propriétés aspectuelles du passif. In Lagae, V. et alii (éds.), 41-63.

Danell, K.J. (1979). Remarques sur la construction dite causative. Stockholm: Almqvist \& Wiksell International.

Donaldson, W.D. (1973). French reflexive verbs : a case grammar description. The Hague, Paris : Mouton.

François, J. (2001). Désémantisation verbale et grammaticalisation, (se) voir employé comme outil de redistribution des actants. Syntaxe \& Sémantique, $2: 159-175$.

Frei, H. (2011) La grammaire des fautes, Presses Universitaires de Rennes.

Gaatone, D. (1983). Le désagréable en syntaxe. Revue Romane, 18 : 161-174.

Haspelmath, M. (1999). Why is grammaticalization irreversible? Linguistics, 37.6: 1043-68.

Helland, H. H. (2002). Le passif périphrastique en français moderne. Copenhague : Museum Tusculanum Press.

Kokutani, S. (2005). Sur l'analyse unie de la construction 'se faire + infinitif en français. In H. Bat-Zeev Shyldkrot \& N. Le Querler (éds.) Les périphrases verbales, Linguisticae Investigationes Supplementa, 25 : 209-227.

Kupferman, L. (1995). La construction passive en se faire. Journal of French Language Studies, 5: 57-83.

Kurylowicz, J. (1965). The evolution of grammatical categories. Esquisses linguistiques, Fink : Munich : 38-54.

Labelle, M. (2002). The French non canonical passive in se faire. In Haraguchi, Shosuke, Bohumil Palek \& Osamu Fujimura (éds.), Proceedings of Linguistics and Phonetics. Tokyo : Charles University Press and Meikai University.

Lagae, V., Carlier, A. \& Benninger, O. (éds.) (2002). Temps et aspect: de la grammaire au lexique. Cahiers Chronos, 10, Amsterdam, New York: Rodopi.

Lagae, V. (2002). Le passif pronominal : une forme complémentaire du passif périphrastique ?. In Lagae, V. et alii (éds.), 133-149.

Lamiroy, B. (1993). Pourquoi il y a deux passifs. Langages, 109 : 53-72.

Le Bellec, C. (2009). La diathèse verbale dans trois langues romanes : vers une description dans le cadre de la Grammaire Fonctionnelle Discursive. Thèse de Doctorat, Université de Toulouse II.

Le Goffic, P. (1993). Grammaire de la phrase française. Paris : Hachette.

Meillet, A. (1912). L'évolution des formes grammaticales. Linguistique historique et linguistique générale. Paris, Champion : 130-148.

Muller, C. (2000). Le passif processif et ses concurrents : définition et quelques particularités. In Schøsler, L. (éd.), 49-69.

Novakova, I. (2008). La construction se faire + Vinf: analyse fonctionnelle. In Représentations du sens linguistique, IV, Helsinki, Finlande.

Schøsler, L. (éd.) (2000). Le passif. Actes du colloque international, Université de Copenhague. 
Spang-Hanssen, E. (1967). Quelques périphrases passives du français moderne. In Actes du $14^{\text {ème }}$ Congrès des Romanistes Scandinaves dédiés à Holger Sten. Revue Romane, $\mathrm{n}^{\circ}$ spécial 1, Copenhague, Akademisk Forlag, 139-147.

Tasmowski, L. \& Van Oevelen, H. (1987). Le causatif pronominal. Revue Romane, $22: 40-58$.

Veecock, C. (2008). Se faire + infinitif : valeurs pragmatico-énonciatives d'une construction 'agentive'. Actes du $1^{\text {er }}$ Congrès Mondial de Linguistique Française, Paris, 2201-2217.

Nombre de mots : environ 8000 